\title{
Imaging of Liver Tumors in Patients with Chronic Liver Disease
}

\author{
Elisabetta Sagrini • Matteo Renzulli • \\ Anna Pecorelli · Federico Stefanini • \\ Fabio Piscaglia
}

Published online: 31 May 2014

(C) Springer Science+Business Media New York 2014

\begin{abstract}
Imaging techniques have become the accepted mainstay for the assessment of liver lesions in cirrhosis and, thanks to the improvement of their diagnostic capabilities in recent years, have further limited the need to resort to bioptic sampling. Hepatocellular carcinoma (HCC) is the most common cause of de-novo liver nodules in cirrhosis, and its diagnosis relies on noninvasive contrast-enhanced imaging studies. Diagnostic criteria have been extensively validated, and the vascular pattern deemed typical for HCC is an arterial hyperenhancement of the nodule followed by washout in the portal or late phase. This pattern provides a positive predictive value for the diagnosis of HCC of about $97 \%$ in nodules in cirrhosis according to the literature. However, the need for a more
\end{abstract}

This article is part of the Topical Collection on Ultrasound in Oncology.

E. Sagrini $(\bowtie)$ - A. Pecorelli · F. Stefanini · F. Piscaglia Division of Internal Medicine, Department of Medical and Surgical Sciences, General and University Hospital S.

Orsola-Malpighi, Via Albertoni 15, 40138 Bologna, Italy e-mail: elisabetta.sagrini2@unibo.it;

elisabetta.sagrini@gmail.com

\section{A. Pecorelli \\ e-mail: anna.pecorelli2@unibo.it \\ F. Stefanini \\ e-mail: fed.stefanini@gmail.com \\ F. Piscaglia \\ e-mail: fabio.piscaglia@unibo.it}

\section{Renzulli}

Division of Radiology, Department of Digestive Diseases and Internal Medicine, General and University Hospital S. Orsola-Malpighi, Via Albertoni 15, 40138 Bologna, Italy e-mail: dr.matteo.renzulli@gmail.com precise differentiation from other malignancies arising in cirrhosis, making up the remaining about $3 \%$, and specifically intrahepatic cholangiocarcinoma, has over time led to changes in the recommendations for the noninvasive diagnosis of HCC in cirrhosis. The present review aims to report recently published interesting studies that have brought new insights into the problem of the characterization and differential diagnosis of liver tumors in chronic liver diseases.

Keywords Hepatocellular carcinoma (HCC) Intrahepatic cholangiocarcinoma (ICC) - Contrastenhanced ultrasonography (CEUS) - Focal liver lesions . Cirrhosis - HCC noninvasive diagnosis

\section{Introduction}

The occurrence of small liver nodules is a frequent event in patients with chronic liver diseases, especially cirrhosis.

Hepatocellular carcinoma (HCC) is the most common de novo liver nodule in cirrhosis; the estimated probability that a nodule in cirrhosis is in fact an HCC is more than $80 \%$ in nodules $\geq 2 \mathrm{~cm}$ in size and between 50 and $75 \%$ in nodules less than $2 \mathrm{~cm}[1 \bullet \bullet, 2,3,4 \bullet]$. A multicenter study on contrast-enhanced ultrasound (CEUS) in focal liver lesions reported frequencies of HCCs, metastases and intrahepatic cholangiocellular carcinomas (ICCs) in cirrhosis of 76.6, 4.3 and $2.5 \%$, respectively. Benign lesions were detected in $14.9 \%$ of cirrhotic livers, with $5.7 \%$ being large regenerative nodules, $2.8 \%$ hemangiomas and $0.3 \%$ adenomas [4 ${ }^{\bullet}$. The characterization of focal liver lesions (FLLs) in liver imaging has to be performed by an operator aware of the clinical setting, and specifically of the presence of any underlying fibrotic chronic liver 
disease, since the range of liver tumors varies widely between patients depending on whether they have diffuse liver disease (including severe fibrosis and cirrhosis) or not.

Since the majority of nodules occurring in cirrhosis are most likely to be HCCs, unless otherwise demonstrated, patients at risk for developing HCC should undergo a semiannual ultrasound-based surveillance program for the early detection of de novo nodules [1••]. According to recently released recommendations, the at-risk population includes cirrhotic patients in either the Child-Pugh stage (in stage $\mathrm{C}$ only if they are eligible for liver transplantation), non-cirrhotic hepatitis B virus (HBV) carriers with active HBV (or inactive but with high viremia or with a family history of HCC), non-cirrhotic patients with chronic HCV and advanced liver fibrosis (stage F3 using the METAVIR classification) and successfully treated patients with chronic HBV or HCV belonging to at risk categories prior to antiviral treatment $[5 \cdot \bullet, 6 \bullet \cdot]$. This aims at an early diagnosis of malignancy that can possibly be cured.

\section{State of the Art}

Imaging of newly occurring nodules in cirrhosis is based on the progressive changes in the blood supply of the nodule during hepatic carcinogenesis. Most HCCs develop from a regenerative nodule through a multistep process of histological dedifferentiation with alterations of the microand macrovasculature [7], progressing from a large regenerative nodule, low-grade dysplastic nodule, highgrade dysplastic nodule and finally to a carcinoma. This process goes along with a progressive loss of normal paired portal and arterial branches and the appearance of newly developed abnormal arterial vessels (neoangiogenesis).

Such changes in the vessel architecture are the key for imaging techniques in diagnosing $\mathrm{HCC}$, whose imaging hallmark is a hypervascular appearance in the arterial followed by washout in the venous phase (Fig. 1) [8•]. Moreover, neoplastic nodules tend to lack reticuloendothelial cells, which is important for characterization by means of contrast agents that have a typical postvascular phase, where malignant nodules stand out as an enhancement defect.

It is widely accepted that the diagnosis of HCC may be obtained noninvasively by means of contrast-enhanced imaging studies. Over time, guidelines have changed their recommendations in this respect.

Updated AASLD (American Association for the Study of Liver Diseases) [1・•] and EASL (European Association for the Study of the Liver) [6••] guidelines now endorse the possibility of diagnosing $\mathrm{HCC}$ in all solid lesions $>1 \mathrm{~cm}$ detected in cirrhosis if they show a typical enhancement pattern (namely arterial hypervascularization and a washout in the venous-delayed phase) at only one imaging modality, including four-phase contrast-enhanced computed tomography (CT) or dynamic contrast-enhanced magnetic resonance imaging (MRI), given the high specificity and positive predictive value (PPV) of this vascular pattern [2, 9]. EASL guidelines, however, have at the same time stipulated that one imaging technique is enough only if performed in centers of excellence with advanced radiological equipment. If an HCC-specific vascular pattern is not seen, the lesion should be assessed by the alternative imaging technique (CT or MRI) or ultimately by biopsy if the imaging findings remain inconclusive. Nodules less than $1 \mathrm{~cm}$ in size are deemed too small to make the diagnosis confidentially and warrant close (3month) follow-ups, looking for possible growth in size. After 1 year without growth, the intervals should be 6 months.

When updating the American and European guidelines, CEUS was excluded from the diagnostic algorithm for the noninvasive diagnosis of HCC in cirrhosis [1••], [6••], [10] for different reasons: CEUS may give false-positive results in case of intrahepatic cholangiocarcinoma (ICC) in cirrhosis [11], and ultrasonographic contrast agents are still awaiting registration in the USA. This last argument indeed conflicts with the ambition of international guidelines to be applicable worldwide.

Nevertheless, there is clear evidence from recent studies that CEUS is indeed a useful primary imaging method in the characterization of HCC and diagnostically comparable to CT/MRI for the diagnosis of a typical vascular pattern of HCC in small $(<2 \mathrm{~cm})$ nodules [2, 3, 12-14]. With regard to detection of a so far unknown nodule, however, the role of CEUS is limited. CEUS is not indicated by guidelines for the detection of HCC nodules, since arterial enhancement can be too short to scan the entire liver in a few seconds, and looking only for a hypoechoic lesion in the late phase can miss HCC lacking typical washout.

B-mode ultrasound remains however the first imaging method indicated for the semiannual surveillance in cirrhotic patients to detect de novo nodules. Guidelines state clearly that nevertheless a CT or MRI-at difference from CEUS, being panoramic techniques-is required for staging purposes to complete the diagnostic workup of HCC nodules before treatment.

The revised AALSD/EASL guidelines are not in agreement with the recommendations of the Asian [15••], Japanese [16••] and World-European Ultrasound Societies (WFUMB-EFSUMB) [17••], which favor including CEUS in the diagnostic algorithm of FLLs in cirrhosis, and the Italian Association for the Study of the Liver (AISF) recommends likewise [5••]. Obviously, more sophisticated MRI and CT are seen as the gold standard and thus mandatory for staging and preoperative treatment planning. 

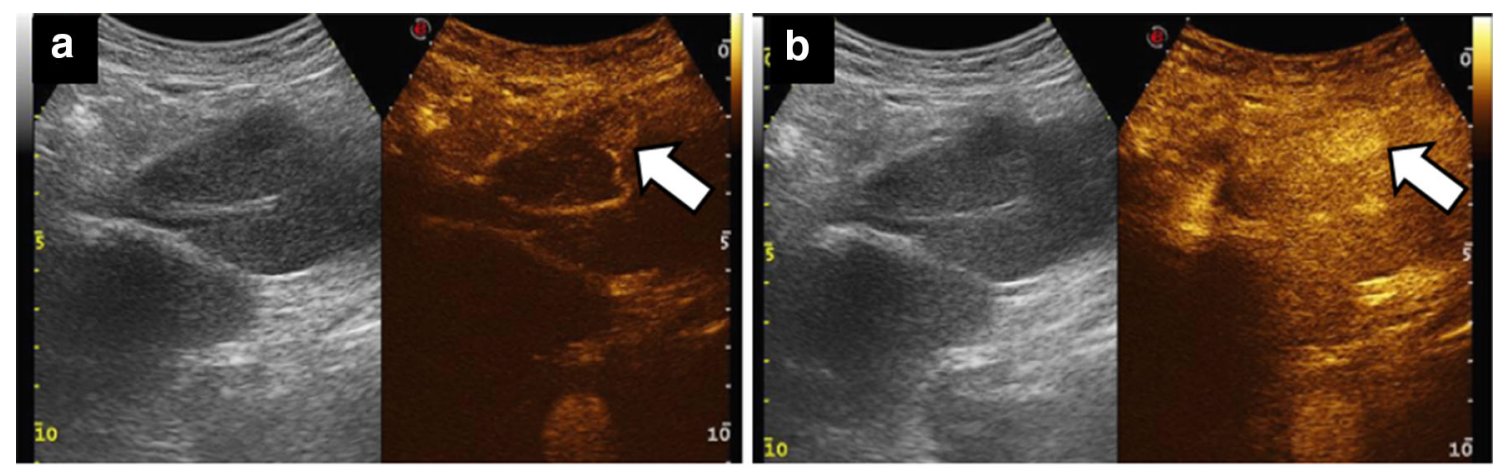

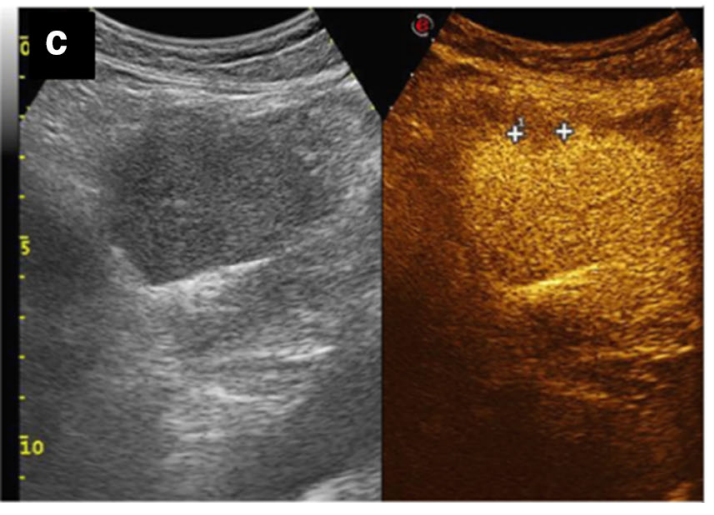

Fig. 1 Typical HCC vascular pattern at CEUS imaging in a cirrhotic patient (G2 on surgical specimen). a Homogeneous arterial hyperenhancement (arrow). b Persistent enhancement in late arterial-early

Imaging Techniques and Contrast Agents for Characterization of Nodules in Cirrhosis

Contrast agents are classified as purely vascular or having an additional post-vascular phase. Second-generation contrast agents for contrast-enhanced ultrasound (SonoVue Bracco SpA, Italy), iodine-based agents for CT and gadolinium chelates for MRI are the most used intravascular agents. SonoVue is the only pure blood pool agent and is quickly cleared through the lungs. Iodine- and gadoliniumbased agents, after having been distributed via the bloodstream, diffuse into the extravascular interstitium, exit tumor capillaries in the venous phase and tend to be retained by fibrous stroma, influencing the late phase appearance of different kinds of tumors. There are two types of postvascular agents. Some agents are taken up by Kupffer/reticuloendothelial cells; one of such agents in ultrasound is Sonazoid, which also has a dynamic vascular phase (GE Healthcare, UK, not yet registered in Western countries); others used in MRI are superparamagnetic iron oxide (SPIO) agents-MRI. The second type is taken up by hepatocytes and then excreted into the bile. There is no such agent in ultrasound. Gadoxetic acid (Gd-EOB-DTPA; Primovist ${ }^{\circledR}$, Bayer Healthcare, Germany) or gadobenate dimeglumine (Gd-BOPTA; Multihance ${ }^{\circledR}$, Bracco, Italy) are commercially available MRI contrast agents of this type.

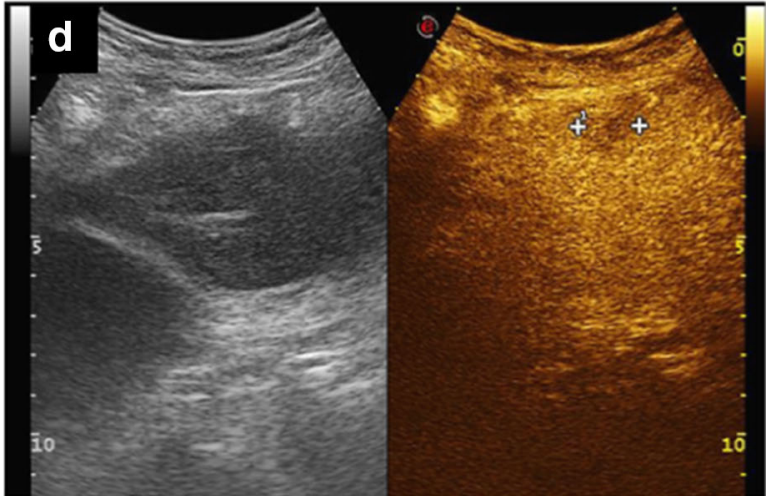

portal phase (34 s post injection; arrow). c, d Late phase hypoenhancement, at 125 and $340 \mathrm{~s}$ post injection, respectively (mild, late washout)

Typically, images are acquired $10-20 \mathrm{~min}$ post Gd-EOBDTPAinjection. Gd-BOPTA has a lower uptake rate and needs 120 min p.i. to accumulate sufficiently in the hepatocytes in order to achieve a diagnostic signal alteration of healthy liver tissue.

There is general agreement between Eastern and Western guidelines on the diagnosis of typical hypervascular HCC in cirrhosis, but Western guidelines consider only the findings provided by vascular phases. AASLD and EASL guidelines include only quadriphasic CT or dynamic MRI for HCC diagnosis, while APASL (Asian Pacific Association for the Study of the Liver) and Japanese guidelines also accept CEUS [15••, 16••]. Eastern guidelines (APASL and Japanese) do not stratify nodule workup with respect to their size and propose a different diagnostic algorithm for the hypovascular nodules in cirrhosis, highlighting the role of postvascular phases of MRI and CEUS with Sonazoid.

\section{Aim}

The purpose of this review is to report recently published interesting studies that have provided new insights into the problem of the characterization and differential diagnosis of liver tumors in chronic liver diseases in the cirrhotic stage. 

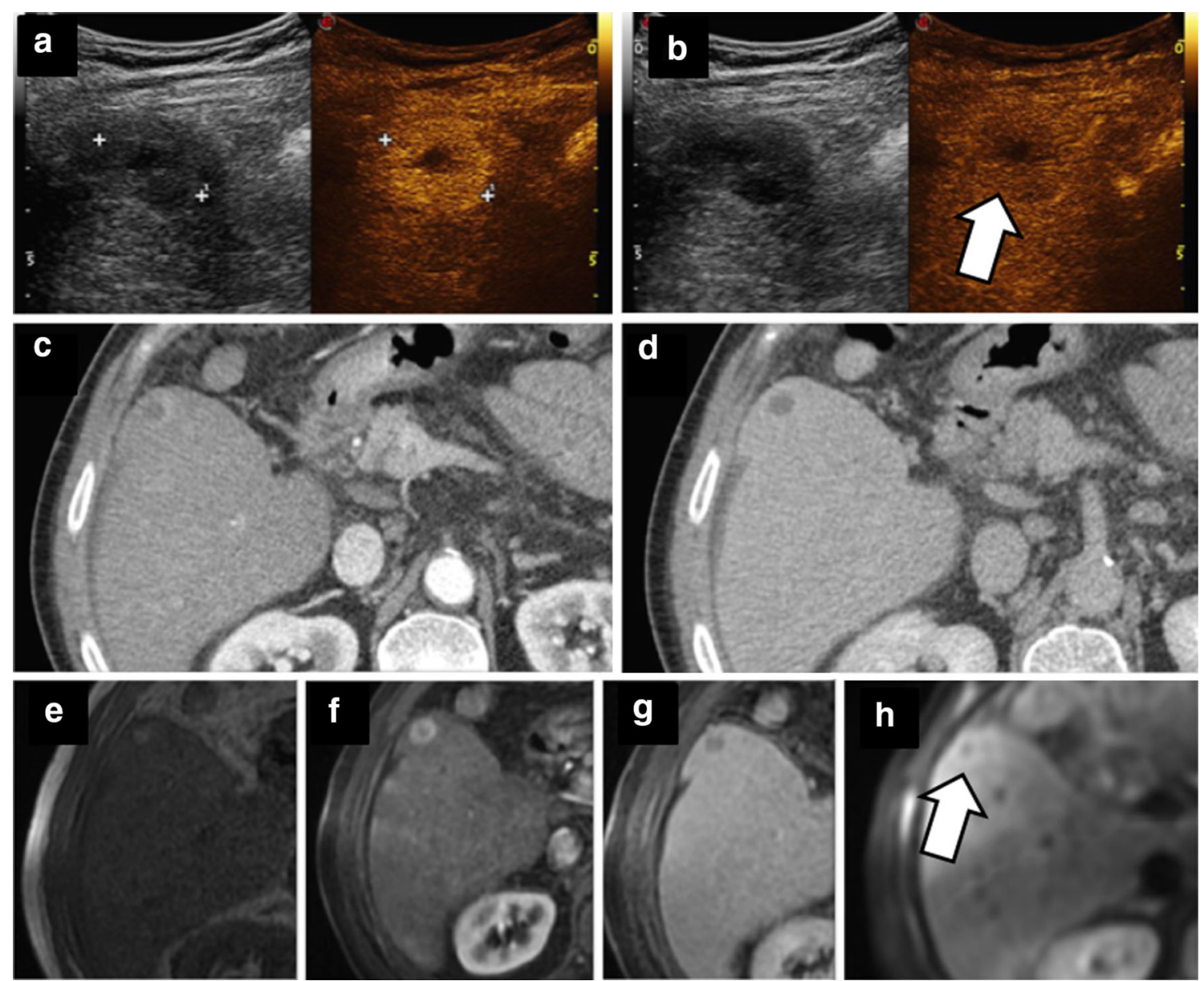

Fig. 2 Hypervascular HCC (G2) of the right liver lobe, with a necrotic central part, in a 64-year-old male with a history of HCVrelated cirrhosis. a CEUS arterial hyperenhancement. The enhancement is mainly homogeneous, and most of the nodule is arterially enhanced, at difference from the rim-like pattern deemed characteristic of ICC. b CEUS shows washout in the late portal phase (110 s) (mild hypoenhancement), with a persistently hypoechoic central portion along all phases (probably necrotic part). c CT: CT scan confirmed the arterial hyperenhancement (hypervascular nodule) with

\section{CEUS in the Diagnosis of HCC}

Typical and Atypical Enhancement Pattern of HCC in CEUS

According to the EFSUMB guidelines published in 2013 [17••], typical CEUS features for HCC are marked arterial enhancement (stronger than in the surrounding parenchyma) followed by washout in the portal/late phase. Arterial enhancement is usually homogeneous (Fig. 1), although larger nodules may enhance heterogeneously (Fig. 2). A rim-like appearance is deemed atypical for HCC [17••], although one study reported $35 \%$ of HCCs to enhance in a rim-like fashion, particularly in larger nodules [18].

Requiring the CEUS appearance to be completely typical will make the technique poorly sensitive, with

the exception of a small, central, persistently hypodense area (probably necrotic area). d The nodule is isodense in late phase, with a slight increase in the hypodense central area. e T1-weighted inphase imaging on MRI shows a hyperintense nodule. f EOB-DTPAenhanced MRI during the arterial phase confirmed the subcapsular nodule with hyperenhancement except for a small central hypovascular area. $\mathbf{g}$ Incomplete MRI washout in delayed phase. $\mathbf{h}$ Hyperintensity on diffusion-weighted MRI imaging

sensitivities as low as $26 \%$ in very small nodules $(<2 \mathrm{~cm})$ [2]. Notably, the lesions missed are more frequently lowgrade HCC than high grade. Iavarone et al. [19] showed that no more than $13 \%$ of small $(\leq 2 \mathrm{~cm})$ G1 HCCs were characterized on radiological exams (any combination of two dynamic, contrast-enhanced modalities) compared to $>50 \%$ of a higher (G2-3) grade HCCs of similar size.

This is due to the fact that well-differentiated HCCs tend to lack the washout in the venous phase at all imaging techniques, but particularly with CEUS (Fig. 3) [3, 19-21]. Therefore, the question whether arterial hyperenhancement alone is sufficient for HCC diagnosis was addressed by some recent publications $[3,21]$.

It is worth remembering that in CT a washout is considered a necessary condition for making the diagnosis of $\mathrm{HCC}$ with confidence. The rationale is to lower the rate of 
Fig. 3 HCC in cirrhosis

displaying a typical homogeneous hypervascularity at CEUS, but with very late and mild washout. a,

b Characteristic CEUS arterial homogeneous

hyperenhancement. c CEUS isoenhancement in portal-late phase $(2 \min 24 \mathrm{~s}$ post injection). d Slightly hypoechoic portion in the nodule appears in very late phase (washout)
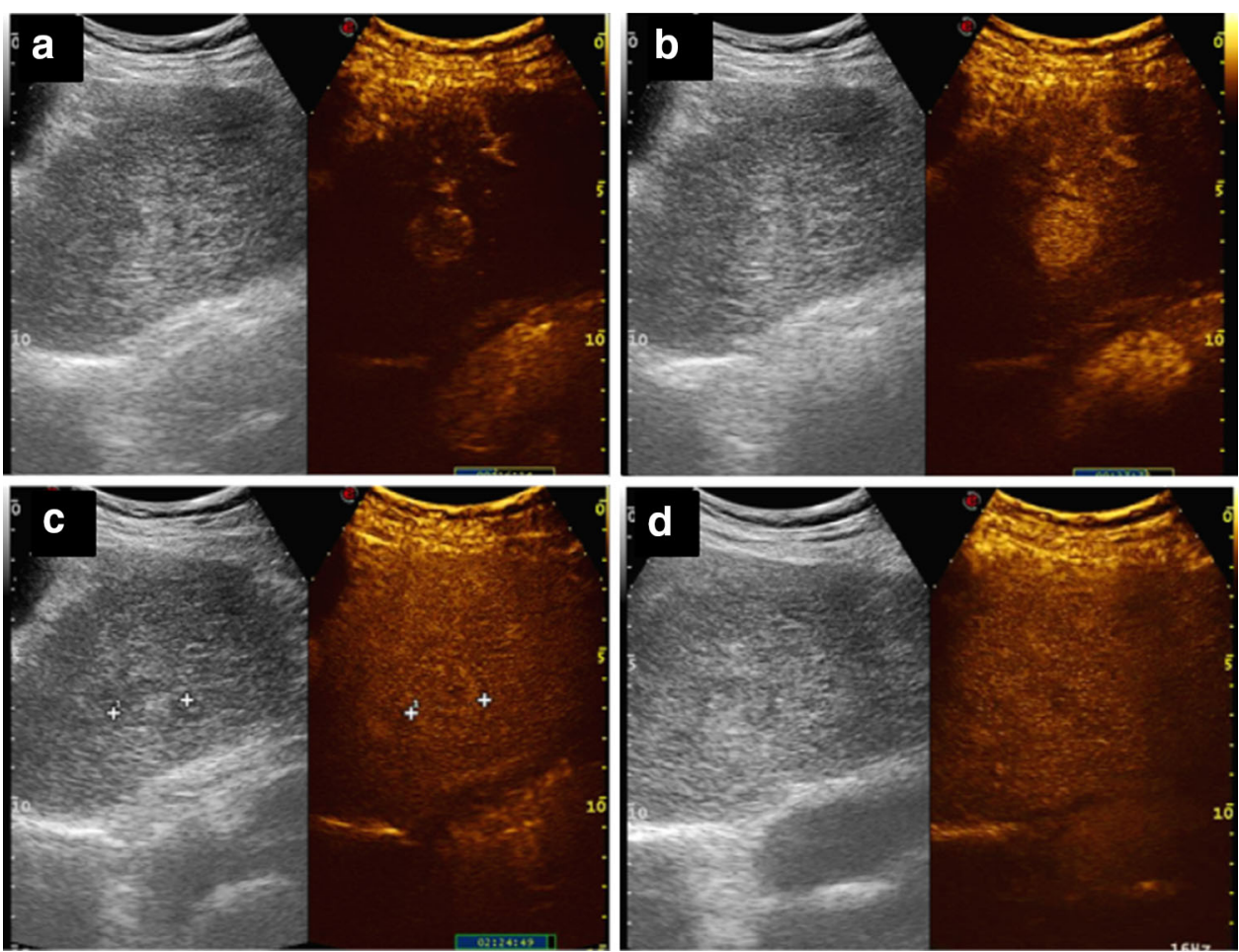

false positives, i.e., perfusion abnormalities falsely diagnosed as HCC. With CEUS, the setting is different, since it is used to characterize suspicious lesions already visible in B-mode or in an accompanying other imaging modality. Thus, the likelihood of a pure perfusion abnormality without any morphological correlate is small.

In a study by Jang et al. [22], CEUS reached an accuracy of $93.2 \%$ for diagnosing an HCC in a previously detected lesion (small 1-2-cm HCCs) based solely on the arterial enhancement pattern. At the same time, all hemangiomas were diagnosed correctly.

Leoni et al. [3] report on 127 primary or recurrent HCC nodules (size 1-3 cm) in which they found an arterial hyperenhancement in $77.2 \%$ of HCCs, with a washout pattern in $41 \%$ of HCCs. Using the classical combination of arterial enhancement and portal/late phase washout (in the following termed the "hyper-hypo pattern") as a criterion for diagnosing $\mathrm{HCC}$, the PPV was $98 \%$, and the accuracy was $51 \%$. An arterial hyperenhancement followed by isoenhancement in portal/late phase was noted in $36.2 \%$ of HCCs. When including this pattern as another criterion for HCC, the resulting overall PPV and accuracy were 94 and $77 \%$, respectively. A persistently isoechoic appearance was noted in $22.8 \%$ of all HCCs, with a higher rate in recurrent nodules (36\%) than in primary HCCs $(13 \%)$. The recurrences were also slightly smaller than for primary lesions (mean size, 18.7 vs. $21.5 \mathrm{~mm}$ ). The authors concluded that the risk that a lesion is an HCC, although typical patterns are missing, is higher in recurrent nodules.
They also confirmed that the occurrence of the typical pattern is strongly influenced by nodule size, as a typical hyper-hypo pattern was found in only $29 \%$ of HCCs $10-20 \mathrm{~mm}$ in size and in $50 \%$ of HCCs $20-30 \mathrm{~mm}$ in size.

Almost all authors have observed that the rate of seeing a typical HCC pattern at CEUS differs from those using CT or MRI. This is related to the washout rather than the arterial hypervascularity $[3,13]$. The typical vascular profile of HCC has been described and validated using extracellular contrast media in dynamic imaging; it is thus related to the specific pharmacokinetics of different contrast agents for different imaging techniques. CT or MRI more often shows a late washout in HCC nodules than CEUS. The opposite is true for intrahepatic cholangiocarcinoma (ICC) in which an early washout is more frequently observed at CEUS than with CT or MRI. This difference in different tumor entities is probably related to the different kinetics of contrast agents and tissue compositions. Sonovue is a pure blood agent that is cleared in a few minutes after injection from the blood pool through the lungs and does not leak out from the vessels. Conversely, the ICC stroma appears to retain CT or MRI contrast agents, which diffuse into the interstitium leaking out from the capillaries, leading to a more persistent enhancement during portal/late phase $[11,23]$. This is a notorious pitfall of missing an ICC, especially for less experienced radiologists.

Timing of washout at CEUS may differ according to cellular differentiation of HCC nodules. Jang et al. demonstrated that poorly differentiated HCCs have an earlier 
(mostly within $90 \mathrm{~s}$ ) washout than better differentiated ones. Of interest, the time of washout in HCCs was independent of whether cirrhosis was present or not [21].

Similar data were recently confirmed by a quantification software analysis study that showed earlier washout and shorter peak arrival time in more aggressive HCCs (with higher intratumoral VEGF expression and neovascular generation), suggesting this could be of prognostic importance [24].

Boozari et al. [20] evaluated CEUS in the grading of hypervascular HCCs according to the presence of late washout. After arterial hyperenhancement, $78 \%$ of higher grade (G2-G3) HCCs showed a washout in the portal/late phase, while lower grade (G1) HCCs showed a washout in only $22 \%$ of cases. In the group of higher grade nodules, the percentage of HCCs $<2 \mathrm{~cm}$ was lower than in the G1 group.

\section{The Guidelines Debate and Potential of CEUS}

CEUS was part of the imaging modalities for the noninvasive diagnosis of HCC in Western countries until 2011 [25], when it was excluded from the revised guidelines [1••, $6 \bullet \bullet$. The rationale for this exclusion, besides the unavailability of microbubble contrast agents in the USA, was the risk of mistaking an ICC for an HCC with CEUS based on a single, small, retrospective study from Vilana et al. [11].

Concerns were raised about whether CEUS has to be completely excluded from the diagnostic algorithm for HCC or if only its role has to be redefined. The AASLD recommendations were not well received in Europe and Asian countries, where CEUS is still included in the HCC guidelines $[5 \bullet \bullet, 15 \bullet \bullet, 16 \bullet \bullet, 17 \bullet \bullet]$.

- CEUS has been maintained in the Japanese guidelines, which endorse separate algorithms for hypo- and hypervascular nodules in cirrhosis [16••]. For arterial hypervascular nodules detected on CT, MRI or CEUS followed by washout in the portal/late phase or no uptake in postvascular phases (Sonazoid-CEUS or EOB-MRI), a diagnosis of typical HCC is accepted. For hypovascular nodules, imaging analysis by means of Sonazoid-CEUS or EOB-MRI is recommended: if the absence of postvascular uptake is confirmed on both, a diagnosis of well-differentiated HCC is made, provided a hemangioma has been ruled out by the typical dynamic phase pattern.

- APASL recommendations state that CEUS is as sensitive as dynamic CT or MRI in the diagnosis of $\mathrm{HCC}$, which can be diagnosed if the classical pattern is shown on dynamic CT, MRI or CEUS. For hypovascular nodules, a lack of uptake on SPIO-MRI or
Sonazoid CEUS in the postvascular phase will confirm an $\mathrm{HCC}[15 \bullet \bullet$.

- The WFUMB/EFSUMB guidelines support the use of CEUS, which can be performed immediately after the detection of a nodule in cirrhosis, allowing a rapid diagnosis [17••, 26]. CT or MRI is however required for a proper pretreatment staging.

- Italian (AISF) recommendations [5••] consider the available scientific evidence insufficient to remove CEUS from the diagnostic tools since a CEUS pattern typical for $\mathrm{HCC}$ has a positive predictive value $>95 \%$ [2], and ICCs represent a minority of instances among all new nodules arising in cirrhosis (1-2\%). Furthermore, not even half of ICCs show a typical HCC pattern at CEUS [11, 27•, 28]. AISF recommendations emphasize the specificity of CEUS in the depiction of a pattern of malignancy (hallmark washin-washout), which, unless concurrent with a highly discordant CT or MRI pattern (rim-like/absence of washout or increasing contrast uptake in the late phase) or with very early $(<60 \mathrm{~s})$ and marked venous washout, indicates an HCC. Although CT or MRI will always be needed for staging purposes [26], the most costeffective approach may be to use CEUS in case of an inconclusive pattern at CT or MRI [5].

\section{Overview of CT and MRI for HCC Characterization in Cirrhosis}

Either dynamic CT or MRI is considered the gold standard for HCC diagnosis and staging in Western guidelines.

However, previous reports indicated that the diagnostic performance of enhanced MRI was better than that of dynamic CT even for only assessing dynamic phases, especially for small nodules [14]. Sensitivities of dynamic CT and MRI for nodules less than $2 \mathrm{~cm}$ ranged between $47-56 \%$ and 63-84\%, respectively [29, 30]. Even in smaller nodules $(<1.5 \mathrm{~cm})$, dynamic MRI performed better than dynamic CT, with reported sensitivities of 75-81\% versus $56 \%$, respectively [31].

Gadoxetic acid (EOB-MRI) and Gd-BOPTA, extracellular agents with hepatobiliary kinetics, allow the exploration of both the vascular pattern and hepatocyte function. These agents are only taken up by normally functioning hepatocytes; malignant nodules therefore present as an enhancement defect during the postvascular hepatocellular phase [32, 33].

The diagnostic yield of these contrast agents has been acknowledged by the Japanese Society of Hepatology and Liver Cancer. The last consensus of the 48th annual meeting of the Liver Cancer Study Group of Japan 
recommended Gd-EOB-DTPA MRI as a first imaging method after the detection of a nodule in cirrhosis and supported the use of dynamic CT only when MRI is not available $[34 \cdot \bullet]$.

The accuracy of Gd-EOB-DTPA-MRI in the characterization of HCC is reported to be superior to both dynamic $\mathrm{CT}$ and MRI with purely extracellular Gd-contrast agents with an overall sensitivity of $72-99 \%, 63-88 \%$ and 58-93\% for EOB-MRI, dynamic MRI and CT, respectively [31, 35-41].

The diagnostic challenge of nodules in cirrhosis is mostly represented by the high rate of small HCCs without a typical vascular pattern, particularly without arterial enhancement. The incidence of atypical nodules in cirrhosis is high, ranging from 28 up to $33 \%$ in selected populations (uncertain and/or discrepant features at CEUS and CT) [39, 42], with about one third being malignant, suggesting that dynamic imaging alone may be limited for an early diagnosis.

MRI with Gd-EOB-DTPA will detect either typical hypervascular HCC or early stage hypovascular HCC, but in the latter instance, MRI alone is insufficient to establish the diagnosis, although the diagnosis of HCC at a very early stage permits the benefit of a timely and minimally invasive treatment.

To this end, the new diagnostic algorithm proposed by the latest Japanese consensus [34] proposed a modified diagnostic workup of nodules in cirrhosis. If a nodule washes out in the late phase after an arterial hypervascularity on EOB-MRI, a diagnosis of HCC is made. If a lesion is hypervascular in the arterial phase and hypointense in the hepatospecific phase, it also has to be considered as HCC, even if no definite washout in the late phase is seen. Obviously, hemangioma needs to be ruled out safely.

In lesions that are hypointense nodules in the hepatospecific phase but show no arterial hypervascularity on dynamic MRI, CEUS with Sonazoid may be used to show either arterial hypervascularity or a filling defect in late phase-both regarded as diagnostic for HCC. Remember that CEUS is more sensitive for arterial enhancement than CT or MRI.

This latter diagnostic algorithm is not applicable in Europe as Sonazoid is not available in Western countries. However, the need has emerged to predict the malignant potential of hypointense nodules on hepatospecific phase at EOB-MRI and obviously lacking the pattern of hemangioma in dynamic phases, which are at this time to be considered at higher risk of progressing to overt HCC.

In keeping with this prognostic information, Kogita et al. [43] showed that EOB uptake decreases already at an early stage of hepatocarcinogenesis, followed by reduction of portal flow. This means that a hypointensity in the hepatospecific phase may occur earlier than delayed vascular phase washout and arterial hypervascularization. Moreover, the degree of hypointensity increased in parallel with the dedifferentiation from dysplastic nodules to HCC.

It is controversial, however, whether dysplastic nodules can be discriminated from HCC on the hepatospecific phase alone. MRI may provide useful information for the treatment and follow-up strategy, being able to provide predictive features of hypervascularization of high-risk nodules.

Kumada et al. [44] demonstrated that a threshold of $15 \mathrm{~mm}$ in size might be critical for the vascularization of hypointense nodules, since nodules at least $15 \mathrm{~mm}$ in size more often progressed to hypervascular HCC (43\% at 6 months and $77 \%$ at 12 months) compared to smaller nodules $(<15 \mathrm{~mm})$, which progressed to typical HCC at a smaller percentage (17\% at both 6 and 12 months).

Hypovascular nodules are characterized by smaller size $(87 \% \leq 2 \mathrm{~cm})$ and vaguely nodular appearance and histopathologically by the absence of vascular invasion, infrequent capsule formation or infiltration, and good differentiation [42].

Other predictive factors for progression to hypervascularity of nodules that are hypointense in the hepatobiliary phase (occurring in 31-32\% in about 6 months) demonstrated by the very recent literature were the presence of fat within the nodule, hyperintensity on T1- and/or T2weighted images, and growth rate (tumor volume doubling time, 542 days) [45, 46].

Choi et al. [47] noted the absence of the typical imaging pattern on dynamic imaging in $28 \%$ of 304 HCCs (31\% in $1-2 \mathrm{~cm}$ in size), the most frequent atypical patterns being the absence of an arterial enhancement and the presence of hypointensity in the hepatospecific phase. The authors suggested that the hepatobiliary phase appearance can be considered an imaging biomarker with prognostic significance as atypical nodules with iso- or hyperintensity in the hepatobiliary phase were smaller, with lower histologic grade and longer time to recurrence than hypointense ones.

With diffusion-weighted imaging (DWI) [48], MRI has gained further diagnostic potential. Differentiation between early HCC and dysplastic nodules relies, according to Western guidelines, on histologic characterization based on immunohistochemistry. DWI differentiates tissues based on the cellular density and may improve the noninvasive characterization, since dysplastic nodules and early HCC differ in the degree of cellular density [49]. Hyperintensity on DWI is regarded as suggestive of HCC (Fig. 2, panel h), and Park et al. [50], applying the enhanced EOB criteria along with hyperintensity on DWI imaging, reached a higher rate of small HCC detection $(82.4 \%)$ than dynamic CT, which showed $53.9 \%$ of HCCs fitting the AASLD criteria. 
Moreover, among atypical (hypovascular or hypervascular without hypointensity) nodules, the hyperintensity on DWI helped with the proper characterization of early HCC.

However, the number of EOB-MRI scans performed in daily practice is limited. MRI is not as widespread as CT, and it takes longer to complete EOB-MRI than dynamic $\mathrm{CT}$. Therefore, the Japanese recommendations suggest the use of quadriphasic $\mathrm{CT}$ at institutions lacking MRI service.

\section{Differential Diagnosis of ICC Versus HCC}

The incidence of peripheral mass-forming ICC is increasing, given the high rates of predisposing conditions such as primary sclerosing cholangitis, as well as chronic $\mathrm{HCV}$ and HBV infection and cirrhosis, which are known risk factors for both hepatocellular and cholangiocellular tumors.

ICC is an infrequent tumor (1-2\% of all liver nodules in cirrhosis), and data about its imaging features are derived from small sample size studies, often retrospectively. Past studies reported heterogeneous enhancement patterns for ICC at CEUS, such as early arterial contrast enhancement followed by marked washout [51, 52], rim-like enhancement, heterogeneous hypoenhancement [18] or absence of enhancement [53].

Most of these studies were not specifically addressed to nodules in cirrhosis, and noninvasive diagnosis is not accepted in patients without cirrhosis. Therefore, a bioptic confirmation will always be necessary.

In the EFSUMB guidelines, rim-like enhancement with hypoechoic central areas during the arterial phase is considered characteristic for ICC as well as an arterial nonenhancement pattern, with hypoenhancement/nonenhancing areas in the portal/venous phase [17••].

Recent studies, specifically targeting populations at risk for primary liver tumors, found that a higher number of ICCs than expected (up to $47 \%$ ) may be hypervascular during arterial phase (Fig. 4), and several studies have reported that small ICCs in cirrhotic patients may show an enhancement pattern similar to those of HCC on CEUS, but occasionally also on CT and MRI [11,54-57].

A recently published study by Chen et al. [27•] compared CEUS enhancement patterns of 50 histologically proven ICCs (5 in a cirrhotic liver) and 50 HCCs (26 in cirrhosis). ICC presented with a peripheral rim in $50 \%$ of lesions (mean size, $7.9 \mathrm{~cm}$ ), heterogeneous hyperenhancement in $20 \%$ (mean size, $7.6 \mathrm{~cm}$ ), heterogeneous hypoenhancement in $24 \%$ (mean size, $6.5 \mathrm{~cm}$ ) and homogeneous hyperenhancement in $6 \%$ (mean size, $3.4 \mathrm{~cm}$ ). All HCC nodules showed an arterial hyperenhancement, heterogeneous in $58 \%$ (mean size, $7.5 \mathrm{~cm}$ ) and homogeneous in $38 \%$ (mean size, $3.9 \mathrm{~cm}$ ). A peripheral rim was observed only in 2 out of 50 nodules
(4\%; mean size, $6.3 \mathrm{~cm}$ ). HCCs showed intratumoral vessels much more often than ICCs. In portal phase, $72 \%$ of ICCs showed a washout, while $24 \%$ remained hypoenhanced in all vascular phases. The study reported a lower risk for CEUS to misdiagnose ICC for HCC than other studies $[11,28]$, with only 5 out of 50 ICC nodules $(10 \%)$ misdiagnosed as HCC. Tumor size heavily influenced enhancement behavior as most ICCs and HCCs less than $3 \mathrm{~cm}$ were homogeneously enhancing, while larger ICCs presented a peripheral rim enhancement mainly due to abundant central fibrous stroma. The diagnostic challenge for CEUS is therefore narrowed to the differential diagnosis of small HCC from ICC presenting with homogeneous enhancement, which is the diagnostic issue most often faced for nodules emerging during ultrasound surveillance of cirrhotic patients.

One study from Vilana et al. [11], which explored ICC patterns at CEUS in cirrhotic livers and based on which the AASLD excluded CEUS from the HCC diagnostic algorithm, was performed on smaller nodules (mean size, $3.2 \mathrm{~cm}$ ); it reported that $48 \%$ of ICCs (10 out of 21 total nodules) displayed an enhancement pattern indistinguishable from those deemed typical for HCC in the AASLD guidelines. This study however did not report an HCC control group, and the sample size was limited.

Bohle et al. [58] retrospectively analyzed the CEUS patterns of 39 HCCs, 11 ICCs (2 with cirrhosis), 3 Klatskin tumors and 4 gallbladder carcinomas. Most HCCs and ICCs showed hyperenhancement during arterial phase (with HCCs more frequently showing complete filling in) followed by late phase hypoenhancement; a hyperenhancement during portal venous phase was observed only in a few HCCs and not in ICCs. Surprisingly, a rim-like pattern was observed in only 3 out of 11 ICCs and in no HCCs. The authors emphasized that no definite consensus exists on the definition of rim-like enhancement. They reported that adhering strictly to a definition of irregular peripheral hyperenhancement (less than $25 \%$ of the whole tumor), with irregular inner stripes extending to the central portions, usually seems to be relatively specific for ICC but that this appears in only some ICCs (Fig. 5).

Among studies that examined enhancement patterns of ICC in cirrhotic livers, Li et al. [59•] performed CEUS on 31 ICCs in chronic liver diseases (mean size, $6.4 \mathrm{~cm}$ ) or cirrhosis (mean size, $5.3 \mathrm{~cm}$ ), and 23 ICCs in normal livers (mean size, $6.2 \mathrm{~cm}$ ). They found that the enhancement was more heterogeneous in ICCs arising in chronic hepatitis/ cirrhosis (60/50\%, respectively) than in those occurring in normal livers (26\%). More ICCs in normal liver had a rimlike enhancement (57\%) than those in chronic hepatitis or cirrhosis $(27 / 31 \%)$. Among patients with cirrhosis, $62.5 \%$ ICCs would have been misclassified as HCC, and most of these were larger than $2 \mathrm{~cm}$. Galassi et al. [28] confirmed 

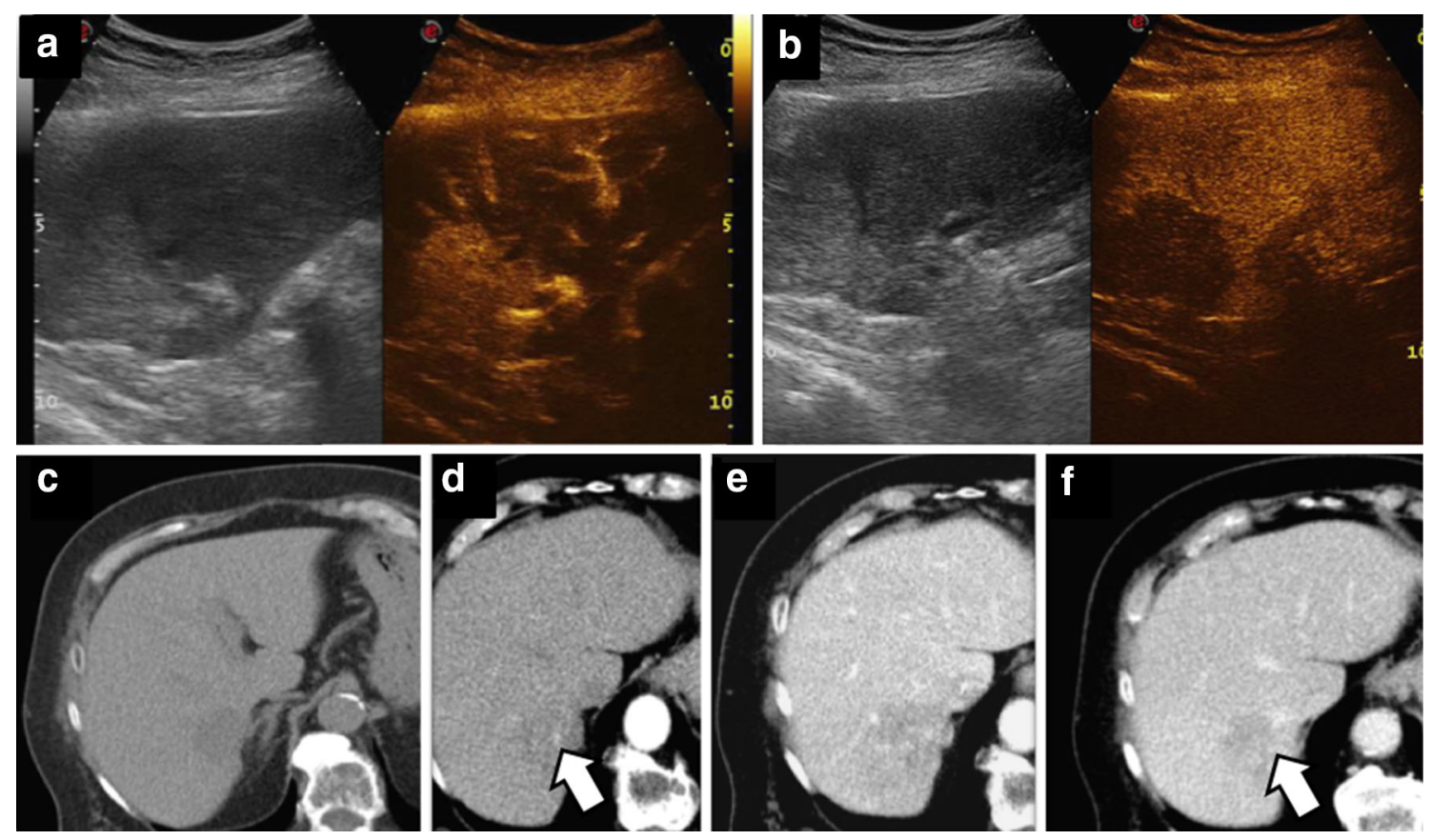

Fig. 4 CEUS appearance of a well-differentiated ICC (G1) of the right liver lobe in cirrhosis. a CEUS: "atypical" homogeneous arterial hyperenhancement. b Marked hypoenhancement (washout) in late phase CEUS. c Nodule is hypodense on pre-contrast CT scans; $\mathbf{d}$ CT: Mild arterial peripheral inhomogeneous enhancement. e CT:

Progressive inhomogeneous enhancement during portal and late phase without clear washout (f), unlike from what is observed at CEUS (washout). The solid mass also demonstrates a "buldging" effect on the liver profile
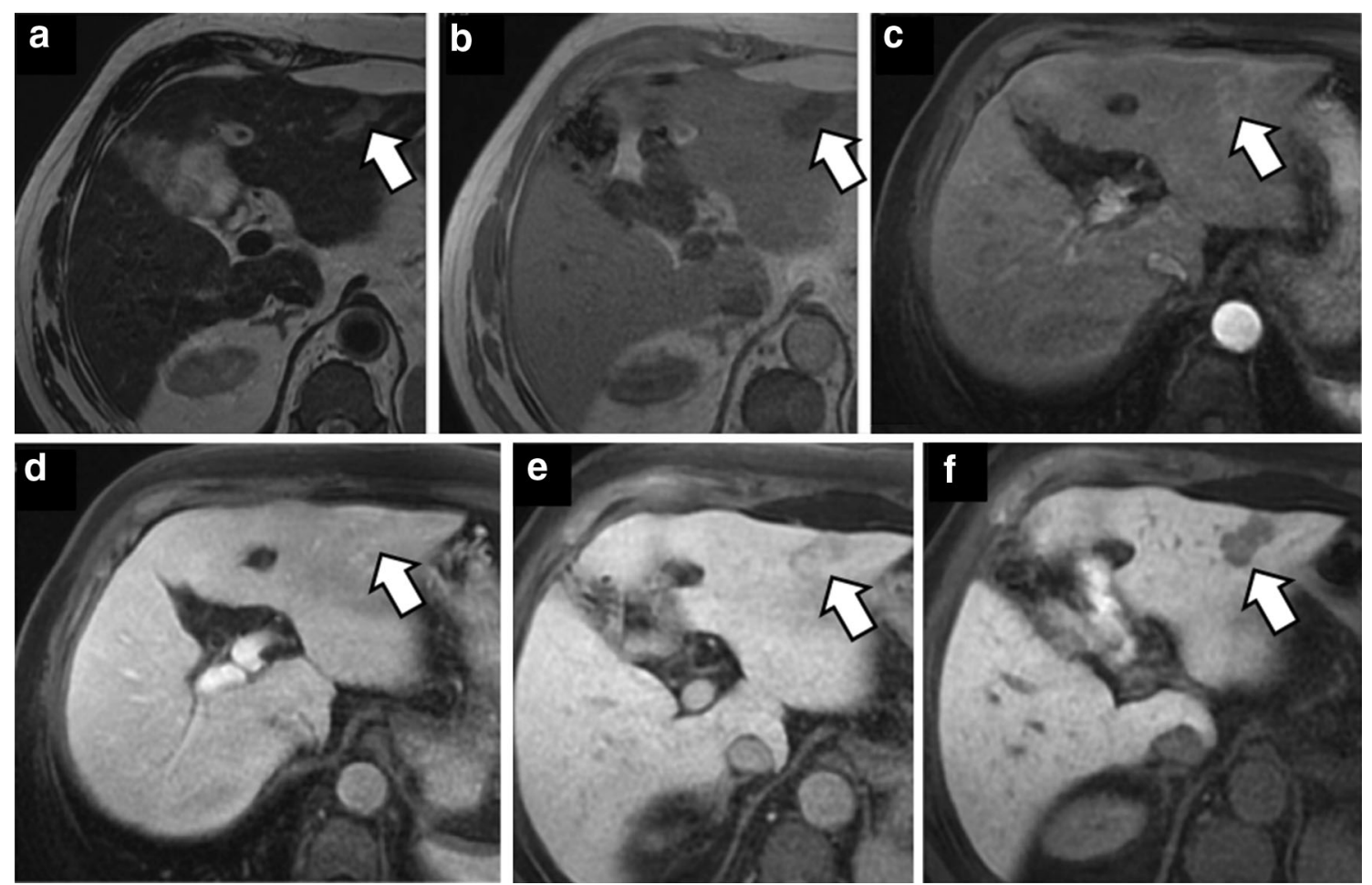

Fig. 5 "Classical" imaging features of ICC (G3) of the left liver lobe in cirrhosis. Gd-EOB-DTPA enhanced MRI appearance. a T2weighted image shows an hyperintense nodule, while hypointensity is demonstrated in T1-weighted in-phase image (b). c Slight

peripheral arterial hyperenhancement. d Isoenhancement on portal phase. e Characteristic late phase "cloud" sign, which has been correlated with central fibrosis (see main text). f Marked hypointensity in the hepatospecific phase 
that a significant (up to $50 \%$ ) proportion of small ICCs in cirrhosis can potentially be mistaken for an HCC because of their similar enhancement pattern in CEUS. A rim-like arterial pattern was seen only in $8 \%$ of ICCs (2 out of 25). However, when the authors considered not only the occurrence of washout in the venous phase, but also its intensity, deeming only a mild washout typical for HCC, the proportion of misclassified nodules decreased to $32 \%$ of all nodules.

Thorough assessment of the intensity of enhancement in liver tumors is a promising tool for increasing diagnostic accuracy of CEUS, especially by means of dedicated software with a time intensity curve (TIC) analysis [60•].

In a study by Chen et al., TIC analysis improved the diagnostic accuracy of CEUS [27•]. The analysis of the intensity of the echo signal in the region of interest put into the tumor and in the adjacent liver parenchyma showed lower intensity and lower slope in the peripheral and central areas of ICCs compared with HCCs. HCCs had richer arterial supply conferring higher intensity in the arterial phase and ICCs washed out to a more marked degree of hypoenhancement during portal/late phase than HCCs.

The introduction of dedicated quantification software may increase reproducibility in comparison to the visual subjective analysis, since it yields semiquantitative perfusion parameters, is able to describe the blood flow pattern and temporal changes in the intensity of enhancement, and may bring more accurate insight to the differential diagnosis of FLLs [61, 62].

Studies have outlined the different contrast-enhancement patterns of ICC in portal/late phase between CEUS and CT or MRI (Fig. 4) due to the different uptakes of the contrast medium from tumoral tissue caused by the different histopathologies of the tumors and interaction with various contrast kinetics $[27 \bullet, 56]$. Delayed phase CT can observe longer enhancement (iso-hyperattenuating appearance) because of the persistent contrast uptake by the fibrous stroma, while a washout appears already in the portal phase at CEUS [11, 63].

Iavarone et al. [23] recently evaluated the contrastenhanced CT appearance of 40 small ICC nodules in a cirrhotic setting (median size, $30 \mathrm{~mm}$ ). Half of the ICC nodules showed a peripheral rim during arterial phase; $42 \%$ of nodules had an increase in contrast enhancement throughout portal/delayed phases, whereas $26 \%$ of nodules showed stable contrast enhancement during a dynamic study ( 2 globally hyperdense, 4 peripherally hyperdense, 4 hypodense in all phases). None of the ICCs presented the vascular pattern deemed typical for HCC, which is a global hyperenhancement followed by washout in the portal/late phase. Most $(74 \%)$ of the rim-like arterial enhancing nodules were $\geq 3 \mathrm{~cm}$ in size, probably because of a central fibrotic scar in larger nodules. The authors confirm the importance of accurate evaluation of delayed phase, as the risk of misdiagnosis with $\mathrm{HCC}$ was ruled out by the isodense pattern observed during the late phase.

A previous study on the CT appearance of ICC in cirrhotic livers [54] reported slightly different results as for washout; few ICCs smaller than $3 \mathrm{~cm}$ (5 out of 8 nodules) showed a washout pattern on portal/venous scans, but the authors did not provide information about their contrast pattern in the delayed phase.

A thorough and prolonged evaluation of enhancement patterns as well as an expertise in the radiological evaluation of nodules in cirrhosis are needed. Nevertheless, CT is not able to establish a definitive noninvasive diagnosis of ICC, not even of malignancy. Conversely, the malignant nature is clearly detected as early/intense washout on CEUS. The combination of such discrepant patterns between CT and CEUS may be the object of future studies.

The use of MRI is gaining widespread consensus for the differential diagnosis between HCC and ICC in cirrhosis.

Frequent MRI imaging findings of ICCs are irregular margins, high intensity on T2-weighted images and low intensity on T1-weighted images (Fig. 5, panel a, b) [64]. On dynamic imaging, they may show peripheral enhancement in the arterial phase followed by centripetal enhancement in portal/delayed phases (Fig. 5, panel c, d). Both peripheral and centripetal enhancement is reported to be more prominent on MRI than on CT [65].

On MRI, Rimola et al. [56] observed a pattern of progressive contrast uptake in the absence of a washout pattern in $80.6 \%$ of ICCs in cirrhotic livers. A progressive enhancement pattern over the subsequent vascular phases was mostly found in larger nodules $(>2 \mathrm{~cm})$ with a stable enhancement pattern in smaller lesions. The authors suggested that if MRI fails to register washout in a nodule in cirrhosis, biopsy should be performed.

The presence of washout in portal and delayed phases varied among studies on MRI, while the introduction of Gd-EOB-DTPA has been reported to increase the diagnostic accuracy of dynamic MRI. Kim et al. [66॰] described $70 \%$ of ICCs showing a typical pattern of arterial peripheral enhancement and progressive centripetal enhancement during portal/delayed phases. The remaining $30 \%$ of ICCs showed an atypical pattern of complete/ partial enhancement during the arterial phase, with complete/partial washout in portal/late phases. They corresponded to an atypical histopathological appearance of ICC, with less fibrotic tissue in the center. On EOB imaging in the hepatobiliary phase, the authors observed a "cloud" sign in $93 \%$ of typical ICCs, namely a persistent enhancement with cloud-like intensity in the center with a peripheral rim defect correlated with central fibrosis (Fig. 5, panel e). Only $33 \%$ of atypical ICCs showed a 
"cloud"-like sign. Thus, atypical, hypervascular ICCs are hardly distinguishable from other malignant and nonmalignant nodules by dynamic vascular behavior alone on CT/ MRI, making biopsy needed again [23].

Interestingly, Xu et al. [67•] showed that the majority of ICCs in cirrhosis displayed strong hypervascular enhancement compared to those in noncirrhotic livers, related to higher arterial and microvessel density.

Findings such as tumor markers, an EOB-cloud appearance, multiplicity and capsule retraction may be helpful anyway, although at present a noninvasive diagnosis of ICC in cirrhosis is still not accepted.

\section{Other FLLs in Cirrhosis}

The prevalence of hepatic metastases from extrahepatic tumors in liver cirrhosis is far lower than that observed in patients without chronic liver disease [68]. These data were confirmed by the DEGUM trial (only $4.3 \%$ of FLLs in cirrhosis were metastases) $[4 \bullet, 69]$. Proposed explanations are liver fibrosis and capillarization, which could create a barrier to tumor cell invasion into the liver parenchyma. This unconsidered, imaging appearance is the same as in healthy livers, with early and marked portal phase washout of the contrast medium irrespective of the arterial enhancement pattern. Clinical background as well as tumor markers play a great supporting diagnostic role.

Primary hepatic lymphoma is more common in HCVHBV-related cirrhosis than in healthy livers and represents up to $1 \%$ of newly developed lesions in cirrhosis [70]. The CEUS appearance has been reported similar to that of a typical HCC, with inhomogeneous arterial hyperenhancement followed by a hypoechoic pattern in the portal/late phase; thus, it should be considered in the differential diagnosis with HCC [71]. However, given its rarity, its impact has been neglected in the guidelines for the management of HCC so far.

\section{Conclusions}

In conclusion, imaging techniques have become the accepted mainstay for the assessment of liver lesions in cirrhosis, and thanks to the improvement of their diagnostic capabilities in recent years, summarized in the present article, have further limited the need to resort to bioptic sampling.

\section{Compliance with Ethics Guidelines}

Conflict of Interest Dr. Elisabetta Sagrini, Dr. Matteo Renzulli, Dr. Anna Pecorelli and Dr. Federico Stefanini each declare no potential conflicts of interest. Dr. Fabio Piscaglia reports personal fees from Bracco, Siemens, GE Healthcare and Esaote, outside of the submitted work.

Human and Animal Rights and Informed Consent This article does not contain any studies with human or animal subjects performed by any of the authors.

\section{References}

References of particular interest have been highlighted as:

- Of importance;

•- Of major importance

1. • Bruix J, Sherman M. Management of hepatocellular carcinoma: an update. Hepatology 2011;53:1020-22. Updated American Association of Liver Diseases (AASLD) guidelines on the management of hepatocellular carcinoma.

2. Sangiovanni A, Manini MA, Iavarone M, et al. The diagnostic and economic impact of contrast imaging techniques in the diagnosis of small hepatocellular carcinoma in cirrhosis. Gut. 2010;59:638-44.

3. Leoni S, Piscaglia F, Granito A, et al. Characterization of primary and recurrent nodules in liver cirrhosis using contrast-enhanced ultrasound: which vascular criteria should be adopted? Ultraschall Med. 2013;34:280-7.

4. - Seitz K, Greis C, Schuler A, et al. Frequency of tumor entities among liver tumors of unclear etiology initially detected by sonography in the noncirrhotic or cirrhotic livers of 1349 patients. Results of the DEGUM multicenter study. Ultraschall Med 2011;32:598-603. The study collects a huge number of focal liver lesions from one of the largest multicentric studies on ultrasound and contrast-enhanced ultrasound in cirrhotic and noncirrhotic patients.

5. •• Bolondi L, Cillo U, Colombo M, et al. Position paper of the Italian Association for the Study of the Liver (AISF): the multidisciplinary clinical approach to hepatocellular carcinoma. Dig Liver Dis 2013;45:712-23. Recently released Italian position paper on the management of hepatocellular carcinoma.

6. •• EASL-EORTC clinical practice guidelines: management of hepatocellular carcinoma. J Hepatol 2012;56:908-43. Updated European (EASL) guidelines on the management of hepatocellular carcinoma.

7. International Consensus Group for Hepatocellular Neoplasia. Pathologic diagnosis of early hepatocellular carcinoma: a report of the international consensus group for hepatocellular neoplasia. Hepatology. 2009;49:658-64.

8. - Matsui O. Detection and characterization of hepatocellular carcinoma by imaging. Clin Gastroenterol Hepatol 2005;3:S136-40. Review collecting mainstay results about sequential changes of imaging findings according to the progression from precancerous nodules to overt HCC.

9. Leoni S, Piscaglia F, Golfieri R, et al. The impact of vascular and nonvascular findings on the noninvasive diagnosis of small hepatocellular carcinoma based on the EASL and AASLD criteria. Am J Gastroenterol. 2010;105:599-609.

10. Forner A, Llovet JM, Bruix J. Hepatocellular carcinoma. Lancet. 2012;379:1245-55.

11. Vilana R, Forner A, Bianchi L, et al. Intrahepatic peripheral cholangiocarcinoma in cirrhosis patients may display a vascular pattern similar to hepatocellular carcinoma on contrast-enhanced ultrasound. Hepatology. 2010;51:2020-9. 
12. Khalili K, Kim TK, Jang HJ, et al. Optimization of imaging diagnosis of 1-2 cm hepatocellular carcinoma: an analysis of diagnostic performance and resource utilization. J Hepatol. 2011;54:723-8.

13. Furlan A, Marin D, Cabassa P, et al. Enhancement pattern of small hepatocellular carcinoma (HCC) at contrast-enhanced US (CEUS), MDCT, and MRI: intermodality agreement and comparison of diagnostic sensitivity between 2005 and 2010 American Association for the Study of Liver Diseases (AASLD) guidelines. Eur J Radiol. 2012;81:2099-105.

14. Quaia E, De Paoli L, Angileri R, et al. Evidence of diagnostic enhancement pattern in hepatocellular carcinoma nodules $\leq 2 \mathrm{~cm}$ according to the AASLD/EASL revised criteria. Abdom Imaging. 2013;38:1245-53.

15. • Omata M, Lesmana LA, Tateishi R, et al. Asian Pacific Association for the Study of the Liver consensus recommendations on hepatocellular carcinoma. Hepatol Int 2010;4:439-74. Updated Asian (APASL) guidelines on the management of hepatocellular carcinoma.

16. $\bullet$ Kudo M, Izumi N, Kokudo N, et al. Management of hepatocellular carcinoma in Japan: Consensus-Based Clinical Practice Guidelines proposed by the Japan Society of Hepatology (JSH) 2010 updated version. Dig Dis 2011;29:339-64. Updated Japanese guidelines on the management of hepatocellular carcinoma.

17. • Claudon M, Dietrich CF, Choi BI, et al. Guidelines and good clinical practice recommendations for contrast enhanced ultrasound (CEUS) in the liver-update 2012: a WFUMB-EFSUMB initiative in cooperation with representatives of AFSUMB, AIUM, ASUM, FLAUS and ICUS. Ultraschall Med 2013;34:11-29. Updated World-European Societies of Ultrasound (WFUMB-EF$S U M B)$ recommendations for CEUS in the liver.

18. Xu HX, Liu GJ, Lu MD, et al. Characterization of small focal liver lesions using real-time contrast-enhanced sonography: diagnostic performance analysis in 200 patients. J Ultrasound Med. 2006;25:349-61.

19. Iavarone M, Sangiovanni A, Forzenigo LV, et al. Diagnosis of hepatocellular carcinoma in cirrhosis by dynamic contrast imaging: the importance of tumor cell differentiation. Hepatology. 2010;52:1723-30.

20. Boozari B, Soudah B, Rifai K, et al. Grading of hypervascular hepatocellular carcinoma using late phase of contrast enhanced sonography: a prospective study. Dig Liver Dis. 2011;43:484-90.

21. Jang HJ, Kim TK, Burns PN, Wilson SR. Enhancement patterns of hepatocellular carcinoma at contrast-enhanced US: comparison with histologic differentiation. Radiology. 2007;244:898-906.

22. Jang HJ, Kim TK, Wilson SR. Small nodules $(1-2 \mathrm{~cm})$ in liver cirrhosis: characterization with contrast-enhanced ultrasound. Eur J Radiol. 2009;72:418-24.

23. Iavarone M, Piscaglia F, Vavassori S, et al. Contrast enhanced CT-scan to diagnose intrahepatic cholangiocarcinoma in patients with cirrhosis. J Hepatol. 2013;58:1188-93.

24. Yang YL, Yang RJ, Liu X, et al. Correlations between the timeintensity parameters of contrast-enhanced ultrasound and clinical prognosis of hepatocellular carcinoma. Clin Imaging. 2013;37:308-12.

25. Bruix J, Sherman M. Management of hepatocellular carcinoma. Hepatology. 2005;42:1208-36.

26. Barreiros AP, Piscaglia F, Dietrich CF. Contrast enhanced ultrasound for the diagnosis of hepatocellular carcinoma (HCC): comments on AASLD guidelines. J Hepatol. 2012;57:930-2.

27. - Chen LD, Xu HX, Xie XY, et al. Intrahepatic cholangiocarcinoma and hepatocellular carcinoma: differential diagnosis with contrast-enhanced ultrasound. Eur Radiol 2010;20:743-53. Study utilizing time intensity curve (TIC) analysis, which can improve the diagnostic accuracy of CEUS especially in the characterization and differential diagnosis of HCC versus ICC.
28. Galassi M, Iavarone M, Rossi S, et al. Patterns of appearance and risk of misdiagnosis of intrahepatic cholangiocarcinoma in cirrhosis at contrast enhanced ultrasound. Liver Int. 2013;33:771-9.

29. Burrel M, Llovet JM, Ayuso C, et al. MRI angiography is superior to helical CT for detection of HCC prior to liver transplantation: an explant correlation. Hepatology. 2003;38:1034-42.

30. Snowberger N, Chinnakotla S, Lepe RM, et al. Alpha fetoprotein, ultrasound, computerized tomography and magnetic resonance imaging for detection of hepatocellular carcinoma in patients with advanced cirrhosis. Aliment Pharmacol Ther. 2007;26:1187-94.

31. Haradome H, Grazioli L, Tinti R, et al. Additional value of gadoxetic acid-DTPA-enhanced hepatobiliary phase MR imaging in the diagnosis of early-stage hepatocellular carcinoma: comparison with dynamic triple-phase multidetector CT imaging. J Magn Reson Imaging. 2011;34:69-78.

32. Choi SH, Lee JM, Yu NC, et al. Hepatocellular carcinoma in liver transplantation candidates: detection with gadobenate dimeglumine-enhanced MRI. AJR Am J Roentgenol. 2008;191:529-36.

33. Fidler J, Hough D. Hepatocyte-specific magnetic resonance imaging contrast agents. Hepatology. 2011;53:678-82.

34. •• Kudo M, Matsui O, Sakamoto M, et al. Role of gadoliniumethoxybenzyl-diethylenetriamine pentaacetic acid-enhanced magnetic resonance imaging in the management of hepatocellular carcinoma: consensus at the Symposium of the 48th Annual Meeting of the Liver Cancer Study Group of Japan. Oncology 2013;84 Suppl 1:21-7. Updated Japanese proposal for a new, simplified diagnostic flow chart for the diagnosis of hepatocellular carcinoma, highlighting the role of Gd-EOB-DTPA MRI.

35. Ichikawa T, Saito K, Yoshioka N, et al. Detection and characterization of focal liver lesions: a Japanese phase III, multicenter comparison between gadoxetic acid disodium-enhanced magnetic resonance imaging and contrast-enhanced computed tomography predominantly in patients with hepatocellular carcinoma and chronic liver disease. Invest Radiol. 2011;45:133-41.

36. Park G, Kim YK, Kim CS, Yu HC, Hwang SB. Diagnostic efficacy of gadoxetic acid-enhanced MRI in the detection of hepatocellular carcinomas: comparison with gadopentetate dimeglumine. Br J Radiol. 2010;83:1010-6.

37. Kim YK, Kim CS, Han YM, et al. Comparison of gadoxetic acidenhanced MRI and superparamagnetic iron oxide-enhanced MRI for the detection of hepatocellular carcinoma. Clin Radiol. 2010;65:358-65.

38. Sano K, Ichikawa T, Motosugi U, et al. Imaging study of early hepatocellular carcinoma: usefulness of gadoxetic acid-enhanced MR imaging. Radiology. 2011;261:834-44.

39. Golfieri R, Renzulli M, Lucidi V, et al. Contribution of the hepatobiliary phase of Gd-EOB-DTPA-enhanced MRI to Dynamic MRI in the detection of hypovascular small $(\leq 2 \mathrm{~cm})$ HCC in cirrhosis. Eur Radiol. 2011;21:1233-42.

40. Marin D, Di Martino M, Guerrisi A, et al. Hepatocellular carcinoma in patients with cirrhosis: qualitative comparison of gadobenate dimeglumine-enhanced MR imaging and multiphasic 64-section CT. Radiology. 2009;251:85-95.

41. Ahn SS, Kim MJ, Lim JS, et al. Added value of gadoxetic acidenhanced hepatobiliary phase MR imaging in the diagnosis of hepatocellular carcinoma. Radiology. 2010;255:459-66.

42. Choi YS, Rhee H, Choi JY, et al. Histological characteristics of small hepatocellular carcinomas showing atypical enhancement patterns on gadoxetic acid-enhanced MR imaging. J Magn Reson Imaging. 2013;37:1384-91.

43. Kogita S, Imai Y, Okada M, et al. Gd-EOB-DTPA-enhanced magnetic resonance images of hepatocellular carcinoma: correlation with histological grading and portal blood flow. Eur Radiol. 2010;20:2405-13.

44. Kumada T, Toyoda H, Tada T, et al. Evolution of hypointense hepatocellular nodules observed only in the hepatobiliary phase 
of gadoxetate disodium-enhanced MRI. AJR Am J Roentgenol. 2011;197:58-63.

45. Higaki A, Ito K, Tamada T, et al. High-risk nodules detected in the hepatobiliary phase of Gd-EOB-DTPA-enhanced MR imaging in cirrhosis or chronic hepatitis: incidence and predictive factors for hypervascular transformation, preliminary results. J Magn Reson Imaging. 2013;37:1377-83.

46. Hyodo T, Murakami T, Imai Y, et al. Hypovascular nodules in patients with chronic liver disease: risk factors for development of hypervascular hepatocellular carcinoma. Radiology. 2013;266: 480-90.

47. Choi JW, Lee JM, Kim SJ, et al. Hepatocellular carcinoma: imaging patterns on gadoxetic acid-enhanced MR Images and their value as an imaging biomarker. Radiology. 2013;267:776-86.

48. Taouli B, Koh DM. Diffusion-weighted MR imaging of the liver. Radiology. 2010;254:47-66.

49. Takayama T, Makuuchi M, Hirohashi S, et al. Malignant transformation of adenomatous hyperplasia to hepatocellular carcinoma. Lancet. 1990;336:1150-3.

50. Park MJ, Kim YK, Lee MH, Lee JH. Validation of diagnostic criteria using gadoxetic acid-enhanced and diffusion-weighted MR imaging for small hepatocellular carcinoma $(\leq 2.0 \mathrm{~cm})$ in patients with hepatitis-induced liver cirrhosis. Acta Radiol. 2013;54:127-36.

51. von Herbay A, Vogt C, Willers R, Haussinger D. Real-time imaging with the sonographic contrast agent SonoVue: differentiation between benign and malignant hepatic lesions. J Ultrasound Med. 2004;23:1557-68.

52. Quaia E, Calliada F, Bertolotto M, et al. Characterization of focal liver lesions with contrast-specific US modes and a sulfur hexafluoride-filled microbubble contrast agent: diagnostic performance and confidence. Radiology. 2004;232:420-30.

53. Nicolau C, Bru C. Focal liver lesions: evaluation with contrastenhanced ultrasonography. Abdom Imaging. 2004;29:348-59.

54. Kim SJ, Lee JM, Han JK, et al. Peripheral mass-forming cholangiocarcinoma in cirrhotic liver. AJR Am J Roentgenol. 2007; 189:1428-34.

55. Chen LD, Xu HX, Xie XY, et al. Enhancement patterns of intrahepatic cholangiocarcinoma: comparison between contrastenhanced ultrasound and contrast-enhanced CT. Br J Radiol. 2008;81:881-9.

56. Rimola J, Forner A, Reig M, et al. Cholangiocarcinoma in cirrhosis: absence of contrast washout in delayed phases by magnetic resonance imaging avoids misdiagnosis of hepatocellular carcinoma. Hepatology. 2009;50:791-8.

57. Kim SA, Lee JM, Lee KB, et al. Intrahepatic mass-forming cholangiocarcinomas: enhancement patterns at multiphasic CT, with special emphasis on arterial enhancement pattern-correlation with clinicopathologic findings. Radiology. 2011;260:148-57.

58. Bohle W, Clemens PU, Heubach T, Zoller WG. Contrastenhanced ultrasound (CEUS) for differentiating between hepatocellular and cholangiocellular carcinoma. Ultraschall Med. 2012;33:E191-5.

59. - Li R, Zhang X, Ma KS, et al. Dynamic enhancing vascular pattern of intrahepatic peripheral cholangiocarcinoma on contrast-enhanced ultrasound: the influence of chronic hepatitis and cirrhosis. Abdom Imaging 2013;38:112-9. First study that compared the CEUS appearance of ICC on cirrhotic versus noncirrhotic livers.

60. - Dietrich CF, Averkiou MA, Correas JM, et al. An EFSUMB introduction into Dynamic Contrast-Enhanced Ultrasound (DCEUS) for quantification of tumour perfusion. Ultraschall Med 2012;33:344-51. The article reviews the recommendation and methodology for quantification of ultrasound images, technical aspects and result interpretation.

61. Quaia E, Palumbo A, Rossi S, et al. Comparison of visual and quantitative analysis for characterization of insonated liver tumors after microbubble contrast injection. AJR Am J Roentgenol. 2006;186:1560-70.

62. Pei XQ, Liu LZ, Liu M, et al. Contrast-enhanced ultrasonography of hepatocellular carcinoma: correlation between quantitative parameters and histological grading. Br J Radiol. 2012;85:e740-7.

63. D'Onofrio $M$, Vecchiato $F$, Cantisani V, et al. Intrahepatic peripheral cholangiocarcinoma (IPCC): comparison between perfusion ultrasound and CT imaging. Radiol Med. 2008;113:76-86.

64. Soyer P, Bluemke DA, Sibert A, Laissy JP. MR imaging of intrahepatic cholangiocarcinoma. Abdom Imaging. 1995;20:126-30.

65. Chung YE, Kim MJ, Park YN, et al. Varying appearances of cholangiocarcinoma: radiologic-pathologic correlation. Radiographics. 2009;29:683-700.

66. - Kim SH, Lee $\mathrm{CH}$, Kim BH, et al. Typical and atypical imaging findings of intrahepatic cholangiocarcinoma using gadolinium ethoxybenzyl diethylenetriamine pentaacetic acid-enhanced magnetic resonance imaging. J Comput Assist Tomogr 2012;36:704-9. The study correlates the MRI appearance of ICCs according to histopathology and highlights the concept of the "cloud" sign in the hepatobiliary phase, which seems to be characteristic of ICC.

67. - Xu J, Igarashi S, Sasaki M, et al. Intrahepatic cholangiocarcinomas in cirrhosis are hypervascular in comparison with those in normal livers. Liver Int 2012;32:1156-64. One of the first studies describing the hypervascular appearance of ICC on cirrhosis versus noncirrhotic livers and correlating it to the histopathology of the different tumors.

68. Seymour K, Charnley RM. Evidence that metastasis is less common in cirrhotic than normal liver: a systematic review of post-mortem case-control studies. Br J Surg. 1999;86:1237-42.

69. Strobel D, Seitz K, Blank W, et al. Contrast-enhanced ultrasound for the characterization of focal liver lesions-diagnostic accuracy in clinical practice (DEGUM multicenter trial). Ultraschall Med. 2008;29:499-505.

70. Caturelli E, Bartolucci F, Biasini E, et al. Diagnosis of liver nodules observed in chronic liver disease patients during ultrasound screening for early detection of hepatocellular carcinoma. Am J Gastroenterol. 2002;97:397-405.

71. Foschi FG, Dall'Aglio AC, Marano G, et al. Role of contrastenhanced ultrasonography in primary hepatic lymphoma. J Ultrasound Med. 2010;29:1353-6. 